\title{
Precipitação média anual e vazão específica média de longa duração, na Bacia do São Francisco ${ }^{1}$
}

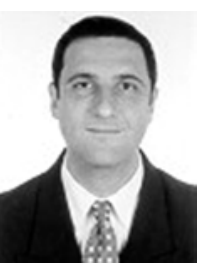

Fernando F. Pruski ${ }^{2}$, Silvio B. Pereira ${ }^{3}$, Luciano F. de Novaes ${ }^{4}$, Demetrius D. da Silva ${ }^{5}$ \& Márcio M. Ramos ${ }^{6}$

\author{
1 Parte do projeto financiado pela ANA/GEF/OEA/PNUMA \\ 2 UFV/DEA. Bolsista do CNPq, CEP 36571-000, Viçosa, MG. Fone: (31) 3899-1912. E-mail: ffpruski@ufv.br (Foto) \\ 3 UFV/DEA. Bolsista do CNPq, Fone: (31) 3899-1925. E-mail: sbueno@vicosa.ufv.br \\ 4 UFV/DEA. Bolsista do CNPq, Fone: (31) 3899-1925. E-mail: Inovaes@vicosa.ufv.br \\ 5 UFV/DEA. Bolsista do CNPq, Fone: (31) 3899-1904. E-mail: david@ufv.br \\ ${ }^{6}$ UFV/DEA. Bolsista do CNPq, Fone: (31) 3899-1914. E-mail: mmramos@ufv.br
}

Protocolo 94 - 19/05/2003 - Aprovado em 12/2/2004

\begin{abstract}
Resumo: O Rio São Francisco abrange uma área de drenagem da ordem de $640.000 \mathrm{~km}^{2}$ e é responsável pela sustentabilidade de 503 municípios situados na bacia, onde vivem cerca de 14 milhões de pessoas. Neste trabalho analisou-se a variação da precipitação média anual e da vazão específica média de longa duração na Bacia do São Francisco. Foram analisados os dados hidrológicos de 178 estações pluviométricas e 77 estações fluviométricas, sendo a vazão específica média de longa duração obtida dividindo-se a vazão média anual pela área de drenagem da estação fluviométrica considerada e a precipitação média em cada área de drenagem, calculada pelo método do Polígono de Thiessen. Os resultados obtidos permitiram as seguintes conclusões: as precipitações diminuem da nascente para a foz do São Francisco, e as vazões específicas médias de longa duração diminuem ao longo do Rio São Francisco, sendo a máxima vazão específica igual a 22,61 $\mathrm{L} \mathrm{s}^{-1} \mathrm{~km}^{-2}$, em Iguatama, primeira estação situada neste, e a mínima, igual a $4,22 \mathrm{~L} \mathrm{~s}^{-1} \mathrm{~km}^{-2}$, em Traipu, última estação desse rio.
\end{abstract}

Palavras-chave: comportamento hidrológico, disponibilidade hídrica, recursos hídricos

\section{Specific yield discharge and mean precipitation in São Francisco Basin}

\begin{abstract}
The São Francisco River drains an area of $640,000 \mathrm{~km}^{2}$ and is responsible for the sustainability of the 503 municipalities in the basin where 14 million people live. This study was undertaken to investigate the mean precipitation and the specific yield discharge in the São Francisco Basin. The analysis was made considering 178 rain-gauge stations and 77 stations to measure stream flow. The specific yield discharge was obtained by dividing the mean annual discharge by the drainage area upstream from the station, and the mean precipitation in the upstream area from each station was obtained by the Thiessen polygon method. The results indicated that the mean precipitation decreased from the Upper São Francisco to Lower São Francisco, and the specific yield discharge decreases along the river, the maximum specific discharge was $22.6 \mathrm{~L} \mathrm{~s}^{-1} \mathrm{~km}^{-2}$, in Iguatama, the first station in the river, and the lowest stream flow was $4.2 \mathrm{~L} \mathrm{~s}^{-1} \mathrm{~km}^{-2}$, in Traipu, the last station in the São Francisco river.
\end{abstract}

Key words: hydrological behavior, water availability, water resources

\section{INTRODUÇÃO}

De acordo com os padrões de disponibilidade e de qualidade da água, seus usos podem se tornar cada vez mais competitivos entre si, resultando na escassez do recurso e em limitações para seu consumo. Ao se considerar os aspectos qualitativos e quantitativos dos recursos hídricos, há que se observar que numa situação de abundância de água ela pode ser tratada como um bem livre, sem valor econômico; entretanto, com o aumento da demanda de água podem ocorrer conflitos entre os usuários.

Os usos da água são divididos em três categorias principais: agrícola, industrial e doméstico. Desses, o setor agrícola é, na maioria dos países em desenvolvimento, o maior usuário. Estima-se, nesses países, que a irrigação utiliza $70 \%$ de toda a água retirada de rios, lagos e mananciais subterrâneos; 
entretanto, o crescimento da demanda de água pelos outros setores, ocasionada pelo crescimento da produção industrial, da urbanização e do aumento da renda das populações, passa a competir com a agricultura irrigada. O setor industrial é responsável por $23 \%$ da retirada de água e o setor urbano por 7\% (Santos, 1998; Cardoso et al., 1998).

Devido ao grande consumo de água na Bacia do São Francisco pela irrigação, qualquer ação que propicie o aumento da eficiência de uso da água na atividade agrícola aumentará expressivamente a disponibilidade de água para os outros usos e mesmo para a ampliação das áreas irrigadas.

A área da Bacia do São Francisco está compreendida entre as latitudes $7^{\circ} 00^{\prime}$ e $21^{\circ} 00^{\prime} \mathrm{S}$ e as longitudes $35^{\circ} 00^{\prime}$ e $47^{\circ} 40^{\prime} \mathrm{W}$, estendendo-se pelos Estados de Minas Gerais, Bahia, Goiás, Pernambuco, Sergipe e Alagoas, além do Distrito Federal. Da nascente, na serra da Canastra, até a foz, no Oceano Atlântico, o Rio São Francisco percorre cerca de 2.700 km, drenando uma área de aproximadamente $640.000 \mathrm{~km}^{2}$, que representa $7,5 \%$ do território nacional (ANEEL, 1998).

As águas do Rio São Francisco atravessam toda a região do "Polígono das Secas", sendo vitais à sustentabilidade e desenvolvimento dos 503 municípios que se situam na Bacia, onde vivem cerca de 14 milhões de habitantes (Almanaque..., 2001).

A Bacia do São Francisco, com disponibilidade de 64,4 bilhões de $\mathrm{m}^{3}$ ano $^{-1}$, responde por $69 \%$ das águas superficiais e por $73 \%$ da disponibilidade superficial garantida do Nordeste. A capacidade total de acumulação de água superficial do Nordeste é de 85,1 bilhões de $\mathrm{m}^{3}$. Desses, 50,9 bilhões, ou seja, $59,8 \%$, se localizam na Bacia do São Francisco (Almanaque..., 2001).

Segundo Lima e Miranda (2001), de 1970 a 1990 a área irrigada na Bacia do São Francisco teve um crescimento da ordem de $286 \%$, o que corresponde a 8.620 ha ano $^{-1}$, enquanto a taxa de crescimento no País foi de $266 \%$.

A Bacia do São Francisco representa, portanto, significativo indutor do desenvolvimento de diversos estados situados na região Nordeste, motivo pelo qual há necessidade de que o comportamento hidrológico da bacia seja devidamente conhecido, para que o aproveitamento de suas águas possa ser otimizado com o menor impacto ambiental possível. Procedeu-se, neste trabalho, à análise das variações espaciais da precipitação média anual e da vazão específica média de longa duração, na Bacia do São Francisco.

\section{MATERIAL E MÉTODOS}

Para a realização do estudo, analisaram-se os dados consistidos de 178 estações pluviométricas e 77 estações fluviométricas pertencentes à rede hidrometeorológica da Agência Nacional de Águas (ANA). Com base na análise dos dados disponíveis, optou-se por selecionar o período-base de 1950-1999 para a realização do estudo.

Após a análise dos dados hidrológicos, àqueles inexistentes ou considerados inconsistentes foram atribuídas falhas para posterior preenchimento. O preenchimento de falhas foi realizado por meio de correlações segundo critérios consagrados de escolha de bases para as regressões.
Para um mesmo curso d'água iniciou-se preferencialmente pela estação mais próxima e quando esta condição não pôde ser atendida, aplicou-se o principio da semelhança hidrológica, tendo nos coeficientes de correlação a base para a tomada de decisão.

Para o preenchimento de falhas de vazão utilizou-se a regressão linear simples:

$$
\mathrm{Y}=\beta_{0} \mathrm{X}+\beta_{1}
$$

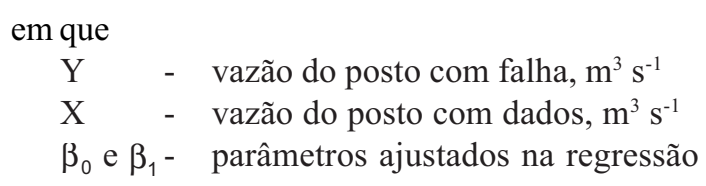

Para o preenchimento de falhas em séries pluviométricas, utilizou-se o método da ponderação regional com base em regressões lineares, que consiste em estabelecer regressões lineares entre os postos com dados a serem preenchidos, $\mathrm{Y}_{\mathrm{c}}$, e cada um dos postos vizinhos, $\mathrm{X}_{1}, \mathrm{X}_{2}, \ldots, \mathrm{X}_{\mathrm{n}}$. De cada uma das regressões lineares efetuadas obtém-se o coeficiente de correlação $\mathrm{r}$, sendo o preenchimento realizado com base na seguinte expressão:

$$
Y_{c}=\frac{r_{y x_{1}} X_{1}+r_{y_{2}} X_{2}+\ldots+r_{y_{x}} X_{n}}{\left(r_{y x_{1}}+r_{y x_{2}}+\ldots+r_{y x_{n}}\right)}
$$

em que

$$
\begin{aligned}
& \mathrm{r}_{\mathrm{yxj}} \text { - coeficiente de correlação entre os postos citados } \\
& \mathrm{n} \text { - número total de postos vizinhos considerados }
\end{aligned}
$$

Para aplicação do método adotou-se como critério mínimo a obtenção de coeficiente de determinação superior a 0,7 e a existência de pelo menos oito pares de eventos entre as estações para a realização da regressão. Nos casos em que a estação em análise apresentou boa correlação com apenas uma estação de apoio utilizou-se, para o preenchimento dos dados de chuva, o preenchimento de regressão linear simples, como foi usado para a vazão.

No caso de estações que apresentavam no mínimo 40 anos de dados, ou seja, pelo menos $80 \%$ da série, não foi feito o preenchimento de falhas.

Para o cálculo da precipitação média na área de drenagem correspondente a cada uma das estações fluviométricas, utilizou-se o método do Polígono de Thiessen, o qual atribui um fator de ponderação aos totais precipitados em cada pluviômetro proporcional à área de influência de cada um. As áreas de influência (pesos) são determinadas no mapa da bacia contendo as estações, unindo-se os pontos adjacentes por linhas retas e, em seguida, traçando-se as mediatrizes dessas retas, formando polígonos. Os lados dos polígonos são os limites das áreas de influência de cada estação (Euclydes et al., 1999).

A precipitação média é calculada pela média ponderada entre a precipitação $\left(\mathrm{P}_{\mathrm{i}}\right)$ de cada estação e o peso a ela atribuído $\left(\mathrm{A}_{\mathrm{i}}\right)$ que é a área de influência de $\mathrm{P}_{\mathrm{i}}$, ou seja: 


$$
P_{m}=\frac{\sum_{i=1}^{n}\left(P_{i} A_{i}\right)}{\sum_{i=1}^{n} A_{i}}
$$

em que:

$\mathrm{P}_{\mathrm{m}}$ - precipitação média na bacia considerada, $\mathrm{mm}$

$\mathrm{P}_{\mathrm{i}}$ - precipitação em cada estação, $\mathrm{mm}$

$\mathrm{A}_{\mathrm{i}}$ - área de influência de $\mathrm{P}_{\mathrm{i}}, \mathrm{km}^{2}$

$\mathrm{n}$ - número de estações pluviométricas consideradas

A vazão específica média de longa duração foi obtida pela divisão dos dados de vazão média anual pela área de drenagem da estação fluviométrica considerada, com base na seguinte equação:

$$
q_{m}=\frac{\bar{Q}}{A_{i}}
$$

em que:

$\mathrm{q}_{\mathrm{m}}$ - vazão específica média de longa duração, $\mathrm{L} \mathrm{s}^{-1} \mathrm{~km}^{-2}$
$\overline{\mathrm{Q}}$ - vazão média anual, $\mathrm{L} \mathrm{s}^{-1}$

$\mathrm{A}_{\mathrm{i}}$ - área de drenagem da estação, $\mathrm{km}^{2}$

Com vistas à representação espacial da precipitação média anual e da vazão específica média de longa duração, foram confeccionados mapas utilizando-se o sistema de informações geográficas ARCVIEW 3.2a. Para tanto, estabeleceram-se intervalos de classe e, a estas, associadas cores que permitam uma visualização melhor dos resultados.

\section{RESULTADOS E DISCUSSÃO}

Na Tabela 1 apresenta-se, para as 77 estações fluviométricas estudadas, o código das estações, a área de drenagem, a precipitação média na sua bacia de drenagem e a vazão específica média de longa duração. As estações foram separadas por sub-bacia, de acordo com a codificação adotada pela ANA, sendo que, para cada sub-bacia, foram identificadas as estações localizadas tanto nos afluentes como no Rio São Francisco.

Evidenciam-se, na Tabela 1, valores de precipitações médias desde $1.506 \mathrm{~mm}$, em Porto do Passarinho (sub-bacia 41), até 847 mm, em Boca da Caatinga (sub-bacia 44). Esta variação foi

\begin{tabular}{|c|c|c|c|c|c|c|c|}
\hline Estação & Código & Sub-bacia & $\begin{array}{c}\text { Área de } \\
\text { Drenagem } \\
\left(\mathrm{km}^{2}\right)\end{array}$ & $\begin{array}{c}\text { Pmédia } \\
(1950-1999)\end{array}$ & $\begin{array}{c}\text { Pmédia na } \\
\text { sub-bacia } \\
(1950-1999)\end{array}$ & $\begin{array}{c}\text { VEM de } \\
\text { longa duração } \\
\left(\mathrm{L} \mathrm{s}^{-1} \mathrm{~km}^{-2}\right)\end{array}$ & $\begin{array}{l}\text { VEM média } \\
\text { na sub-bacia } \\
\left(\mathrm{L} \mathrm{s}^{-1} \mathrm{~km}^{-2}\right)\end{array}$ \\
\hline Vargem Bonita & 40025000 & \multirow{13}{*}{40} & 299 & 1.420 & \multirow{13}{*}{1.386} & 28,19 & \multirow{13}{*}{17,25} \\
\hline Tapiraí & 40060000 & & 543 & 1.430 & & 22,23 & \\
\hline Carmo do Cajuru & 40150000 & & 2.402 & 1.387 & & 15,67 & \\
\hline Jaguaruna & 40300000 & & 1.545 & 1.367 & & 12,85 & \\
\hline Velho da Taipa & 40330000 & & 7.350 & 1.399 & & 13,42 & \\
\hline Estação Álvaro da Silveira & 40400000 & & 1.803 & 1.422 & & 14,13 & \\
\hline São Brás do Sacuí-Montante & 40549998 & & 446 & 1.362 & & 16,93 & \\
\hline Entre Rios de Minas & 40680000 & & 469 & 1.367 & & 18,53 & \\
\hline Belo Vale & 40710000 & & 2.690 & 1.362 & & 17,79 & \\
\hline Alberto Flores & 40740000 & & 3.945 & 1.355 & & 15,34 & \\
\hline Ponte Nova do Paraopeba & 40800001 & & 5.680 & 1.357 & & 14,65 & \\
\hline Ponte da Taquara & 40850000 & & 8.720 & 1.358 & & 14,40 & \\
\hline Barra do Funchal & 40930000 & & 881 & 1.428 & & 20,16 & \\
\hline Iguatama * & 40050000 & \multirow{4}{*}{40} & 4.846 & 1.415 & \multirow{4}{*}{1.388} & 22,61 & \multirow{4}{*}{18,63} \\
\hline Ponte do Chumbo* & 40070000 & & 9.255 & 1.365 & & 18,86 & \\
\hline Porto das Andorinhas* & 40100000 & & 13.087 & 1.384 & & 16,37 & \\
\hline Porto da Barra * & 40102000 & & 14.370 & 1.386 & & 16,66 & \\
\hline Major Porto & 41050000 & \multirow{8}{*}{41} & 1.396 & 1.482 & \multirow{8}{*}{1.345} & 13,66 & \multirow{8}{*}{14,00} \\
\hline Porto do Passarinho & 41075001 & & 4.330 & 1.506 & & 15,62 & \\
\hline Ponte Raul Soares & 41340000 & & 4.780 & 1.347 & & 16,06 & \\
\hline Pirapama & 41600000 & & 7.838 & 1.319 & & 13,12 & \\
\hline Ponte do Licínio & 41650000 & & 10.980 & 1.292 & & 11,89 & \\
\hline Presidente Juscelino & 41780000 & & 3.912 & 1.333 & & 18,47 & \\
\hline Santo Hipólito & 41818000 & & 16.528 & 1.277 & & 12,21 & \\
\hline Várzea da Palma & 41990000 & & 25.940 & 1.206 & & 10,93 & \\
\hline Pirapora-Barreiro * & 41135000 & 41 & 61.880 & 1.349 & 1.349 & 13,68 & 13,68 \\
\hline Porto Aliança & 42090000 & \multirow{4}{*}{42} & 4.374 & 1.034 & \multirow{4}{*}{1.236} & & \\
\hline Santa Rosa & 42395000 & & 12.880 & 1.336 & & & \\
\hline Porto da Extrema & 42690001 & & 29.060 & 1.279 & & & \\
\hline Porto Alegre & 42980000 & & 40.300 & 1.293 & & & \\
\hline
\end{tabular}

Tabela 1. Área de drenagem, precipitação e vazão específica média (VEM) de longa duração para as 77 estações fluviométricas estudadas 
(Continuação da Tabela 1)

\begin{tabular}{|c|c|c|c|c|c|c|c|}
\hline Estação & Código & Sub-bacia & $\begin{array}{c}\text { Área de } \\
\text { Drenagem } \\
\left(\mathrm{km}^{2}\right)\end{array}$ & $\begin{array}{c}\text { Pmédia } \\
(1950-1999)\end{array}$ & $\begin{array}{c}\text { Pmédia na } \\
\text { sub-bacia } \\
(1950-1999)\end{array}$ & $\begin{array}{c}\text { VEM de } \\
\text { longa duração } \\
\left(\mathrm{L} \mathrm{s}^{-1} \mathrm{~km}^{-2}\right)\end{array}$ & $\begin{array}{l}\text { VEM média } \\
\text { na sub-bacia } \\
\left(\mathrm{L} \mathrm{s}^{-1} \mathrm{~km}^{-2}\right)\end{array}$ \\
\hline Montante Barra do Jequitaí * & 42030000 & \multirow{2}{*}{42} & 90.990 & 1.305 & \multirow{2}{*}{1.288} & 12,03 & \multirow{2}{*}{11,64} \\
\hline Cachoeira da Manteiga * & 42210000 & & 107.250 & 1.271 & & 11,24 & \\
\hline Arinos & 43430000 & \multirow[t]{5}{*}{43} & 11.710 & 1.295 & \multirow[t]{5}{*}{1.236} & 12,26 & \multirow{5}{*}{11,83} \\
\hline Vila Urucuia & 43670000 & & 18.600 & 1.266 & & 11,33 & \\
\hline Fazenda Conceição & 43675000 & & 2.200 & 1.162 & & 14,40 & \\
\hline Santo Inácio & 43880000 & & 23.765 & 1.227 & & 10,77 & \\
\hline Barra do Escuro & 43980000 & & 24.658 & 1.228 & & 10,37 & \\
\hline São Romão * & 43200000 & 43 & 154.100 & 1.271 & 1.271 & 10,62 & 10,62 \\
\hline Usina do Pandeiros & 44250000 & \multirow{2}{*}{44} & 3.812 & 1.168 & \multirow{2}{*}{1.008} & 6,54 & \multirow{2}{*}{3,81} \\
\hline Boca da Caatinga & 44950000 & & 30.474 & 847 & & 1,07 & \\
\hline São Francisco * & 44200000 & \multirow{3}{*}{44} & 182.537 & 1.262 & \multirow{3}{*}{1.250} & 11,04 & \multirow{3}{*}{10,54} \\
\hline Pedras de Maria da Cruz * & 44290002 & & 191.063 & 1.250 & & 10,71 & \\
\hline Manga $*$ & 44500000 & & 202.400 & 1.237 & & 9,88 & \\
\hline São Gonçalo & 45131000 & \multirow{11}{*}{45} & 6.186 & 1.229 & \multirow{11}{*}{1.121} & 11,20 & \multirow{11}{*}{9,17} \\
\hline Fazenda Porto Alegre & 45170000 & & 5.730 & 1.151 & & 10,94 & \\
\hline Lagoa das Pedras & 45210000 & & 12.120 & 1.181 & & 11,00 & \\
\hline Capitânea & 45220000 & & 2.196 & 1.140 & & 6,12 & \\
\hline Juvenília & 45260000 & & 15.600 & 1.148 & & 9,54 & \\
\hline Correntina & 45590000 & & 4.075 & 1.008 & & 7,91 & \\
\hline Mocambo & 45740000 & & 8.130 & 958 & & 5,37 & \\
\hline Arrojado & 45770000 & & 5.278 & 1.110 & & 10,90 & \\
\hline Gatos & 45840000 & & 6.867 & 1.182 & & 11,67 & \\
\hline Colônia do Formoso & 45880000 & & 8.695 & 1.182 & & & \\
\hline Santa Maria da Vitória & 45910000 & & 29.570 & 1.044 & & 7,04 & \\
\hline Carinhanha * & 45298000 & \multirow{2}{*}{45} & 251.209 & 1.179 & \multirow{2}{*}{1.165} & 8,87 & \multirow{2}{*}{8,29} \\
\hline Bom Jesus da Lapa * & 45480000 & & 273.750 & 1.150 & & 7,70 & \\
\hline Derocal & 46455000 & \multirow{11}{*}{46} & 6.231 & 1.103 & \multirow{11}{*}{1.040} & 7,83 & \multirow{11}{*}{5,52} \\
\hline Fazenda Coqueiro & 46490000 & & 4.300 & 1.055 & & 1,21 & \\
\hline Fazenda Redenção & 46543000 & & 5.400 & 1.103 & & 8,89 & \\
\hline Barreiras & 46550000 & & 18.560 & 956 & & 5,70 & \\
\hline Nova Vida & 46590000 & & 7.155 & 1.038 & & 6,60 & \\
\hline São Sebastião & 46610000 & & 32.586 & 1.081 & & 5,51 & \\
\hline Taguá & 46650000 & & 35.564 & 1.081 & & 5,02 & \\
\hline Fazenda Macambira & 46675000 & & 39.256 & 1.065 & & 4,47 & \\
\hline Formosa do Rio Preto & 46790000 & & 14.210 & 952 & & 6,52 & \\
\hline Ibipetuba & 46830000 & & 18.200 & 983 & & 4,99 & \\
\hline Boqueirão & 46902000 & & 68.540 & 1.018 & & 3,97 & \\
\hline Paratinga * & 46105000 & & 318.028 & 1.123 & & 7,29 & \\
\hline Ibotirama * & 46150000 & & 325.200 & 1.115 & & 7,39 & \\
\hline Morpará * & 46360000 & 46 & 348.074 & 1.086 & 1.098 & 7,12 & 6,91 \\
\hline Barra * & 46998000 & & 433.280 & 1.068 & & 6,09 & \\
\hline Pilão Arcado * & 47302000 & 47 & 443.100 & 1.044 & 1.044 & 5,90 & 5,90 \\
\hline Juazeiro * & 48020000 & & 510.800 & 984 & & 4,94 & \\
\hline Santa Maria da Boa Vista * & 48290000 & 48 & 530.000 & 966 & 964 & 4,80 & 4,74 \\
\hline Ibó * & 48590000 & & 568.600 & 943 & & 4,49 & \\
\hline Pão de Açúcar * & 49370000 & 49 & 608.900 & 907 & 905 & 4,34 & 4,28 \\
\hline Traipu $*$ & 49660000 & 49 & 622.600 & 903 & 905 & 4,22 & 4,20 \\
\hline
\end{tabular}

(*) Estações localizadas na calha principal do Rio São Francisco

também evidenciada para as vazões específicas de longa duração, sendo observados valores que oscilaram desde $28,19 \mathrm{~L} \mathrm{~s}^{-1} \mathrm{~km}^{-2}$, obtidos em Vargem Bonita (sub-bacia 40), até $1,07 \mathrm{~L} \mathrm{~s}^{-1} \mathrm{~km}^{-2}$, verificado em Boca da Caatinga (sub-bacia 44).
Embora tenham sido observados valores bastante baixos (inferiores a 1,3 $\mathrm{L} \mathrm{s}^{-1} \mathrm{~km}^{-2}$ ) para a vazão específica média de longa duração nas estações Boca da Caatinga e Fazenda Coqueiro, nas demais estações a vazão específica foi maior que $4 \mathrm{~L} \mathrm{~s}^{-1} \mathrm{~km}^{-2}$. 
Para as estações localizadas nos afluentes do São Francisco foram constatados decréscimos da precipitação média e da vazão específica média de longa duração das sub-bacias situadas mais próximas à nascente do São Francisco para as sub-bacias situadas mais próximas à foz, sendo que, enquanto na sub-bacia 40 se tem uma precipitação média de $1.386 \mathrm{~mm}$ e uma vazão específica média de longa duração de $17,19 \mathrm{~L} \mathrm{~s}^{-1} \mathrm{~km}^{-2}$, na sub-bacia 46 a precipitação média é de $1.040 \mathrm{~mm}$ e a vazão específica média de $5,52 \mathrm{~L} \mathrm{~s}^{-1} \mathrm{~km}^{-2}$. A única exceção a esta tendência é a subbacia 44.

Verifica-se, para as vazões específicas médias de longa duração, que a primeira estação situada no São Francisco (Iguatama, localizada na sub-bacia 40 e com área de drenagem de $4.846 \mathrm{~km}^{2}$ ) tem contribuição específica de $22,61 \mathrm{~L} \mathrm{~s}^{-1} \mathrm{~km}^{-2}$, a maior dentre todas as estações situadas no rio principal, enquanto a última estação (Traipu, localizada na sub-bacia 49 e com área de drenagem de $622.600 \mathrm{~km}^{2}$ ) tem vazão específica de $4,22 \mathrm{~L} \mathrm{~s}^{-1} \mathrm{~km}^{-2}$, a menor dentre todas as estações situadas no São Francisco.

Na Figura 1 apresenta-se o mapa com a distribuição da precipitação média anual no período de 1950 a 1999 para as áreas de drenagens das 77 estações fluviométricas estudadas e, na Figura 2, o mapa com a distribuição da vazão específica média de longa duração. São apresentados também, em cada figura, os limites das sub-bacias do São Francisco, segundo a

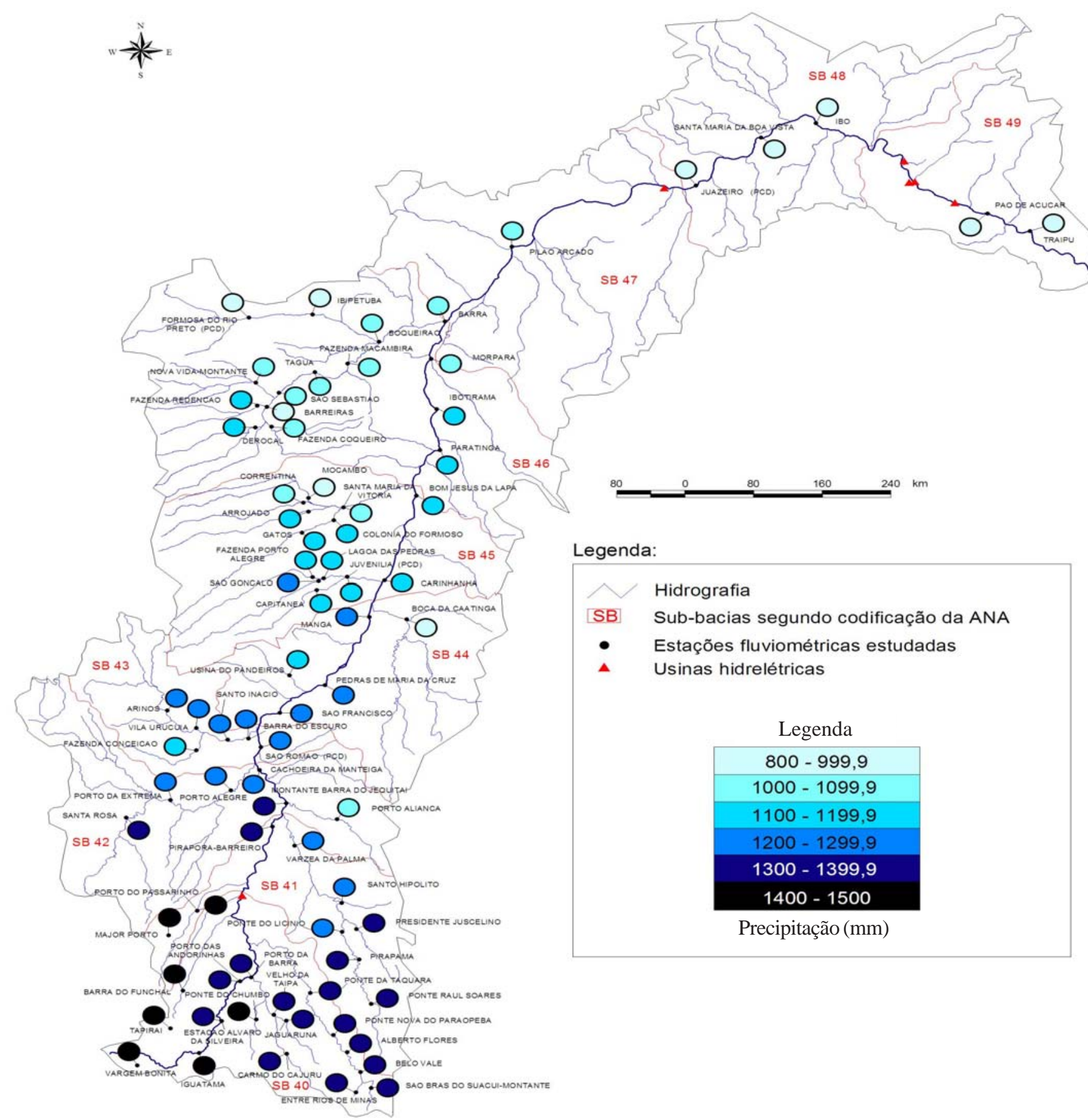

Figura 1. Distribuição da precipitação média anual na Bacia do São Francisco 
codificação da ANA. Evidencia-se, na Figura 1, a redução da precipitação média anual da nascente em direção à foz, sendo que, enquanto as precipitações médias nas áreas de drenagem das estações fluviométricas situadas na sub-bacia 40 são todas maiores que $1.300 \mathrm{~mm}$, para as estações localizadas a partir da sub-bacia 45 as precipitações médias anuais são inferiores a
$1.200 \mathrm{~mm}$. As precipitações médias nas áreas de drenagem das estações situadas nas sub-bacias 48 e 49 variam de 800 a $1.000 \mathrm{~mm}$.

A análise das vazões específicas médias de longa duração (Figura 2) para as estações situadas no Rio São Francisco vem confirmar o comportamento descrito, sendo evidente a redução da vazão específica com o aumento da área de contribuição, ou

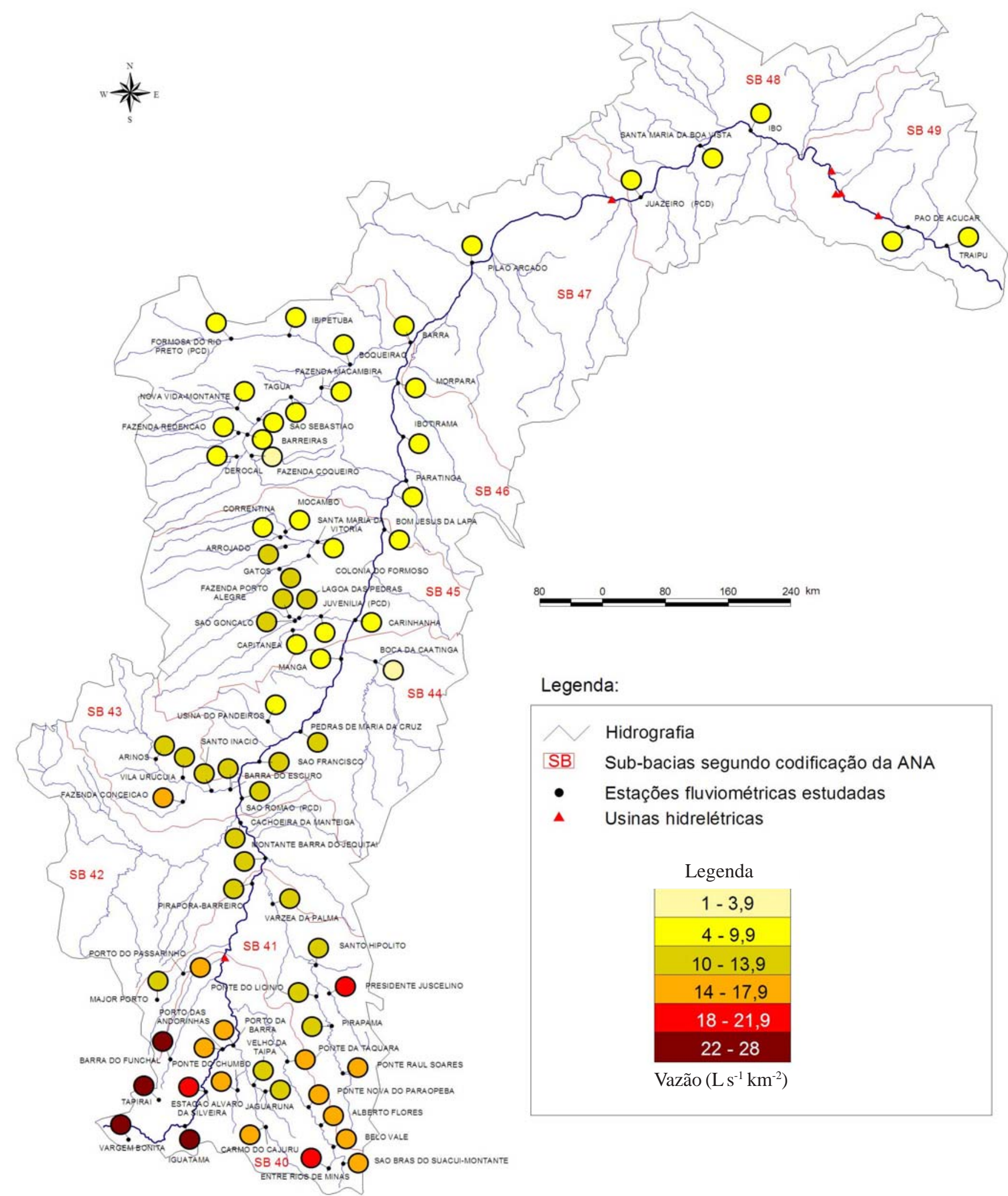

Figura 2. Distribuição da vazão específica média de longa duração na Bacia do São Francisco 
seja, quando do deslocamento das posições próximas à nascente em direção à foz.

\section{CONCLUSÕES}

1. As precipitações diminuem da nascente para a foz do São Francisco, sendo a maior precipitação média anual observada na área de drenagem da estação Porto Passarinho, situada na sub-bacia 40, igual a $1.506 \mathrm{~mm}$, e a mínima precipitação média anual observada na área de drenagem pertinente a Traipu, localizada na sub-bacia 49 igual a $902 \mathrm{~mm}$.

2. As vazões específicas médias de longa duração diminuem ao longo do Rio São Francisco, da sua nascente para a foz, sendo a máxima vazão específica média de longa duração igual a 22,61 L s s $\mathrm{km}^{-2}$, observada em Iguatama (área de drenagem de $4.846 \mathrm{~km}^{2}$ ), primeira estação situada neste, e a mínima vazão específica igual a 4,22 $\mathrm{L} \mathrm{s}^{-1} \mathrm{~km}^{-2}$, evidenciada em Traipu (área de drenagem de $622.600 \mathrm{~km}^{2}$ ), última estação estudada ao longo do São Francisco.

\section{LITERATURA CITADA}

Almanaque Vale do São Francisco 2001. Brasília DF, CODEVASF, 2001.411p.

ANEEL-Agência Nacional de Energia Elétrica. Atlas hidrológico brasileiro - Versão 1.0. Brasília DF, ANEEL, CD. ROM. 1998.

Cardoso, H.E.A.; Mantovani, E.C.; Costa, L.C. As águas na agricultura. In: Agroanalysis. Instituto Brasileiro de Economia. Centro de Estudos Agrícolas. Rio de Janeiro, v.19, n.3, p.27-28, 1998.

Euclydes, H.P., Souza, E. F., Ferreira, P. A. RH $3.0-$ Regionalização hidrológica: manual do programa. Viçosa, MG: UFV/DEA; Brasília, DF: MMA; Belo Horizonte, MG: RURALMINAS, 1999, 149p.

Lima, J.P.R.; Miranda, E.A. Fruticultura irrigada no vale do São Francisco: incorporação tecnológica, competitiva e sustentabilidade. Revista Econômica do Nordeste, Fortaleza, v.32, n. especial, p.611-632, 2001.

Santos, J.R.M. dos. Irrigar é preciso. In: Agroanalysis. Instituto Brasileiro de Economia. Centro de Estudos Agrícolas. Rio de Janeiro, v.19, n.3, p.29-34, 1998. 